\title{
UNIVERSALITY OF CRITICAL-POINT EXPONENTS WITH RESPECT TO LATTICE ANISOTROPY*
}

\author{
G. PAUL ** and H. E. STANLEY *** \\ Department of Physics, Massachusetts Institute of Technology, \\ Cambridge, Massachusetts 02139, USA
}

Received 26 October 1971

\begin{abstract}
The universality hypothesis predicts that critical-point exponents should be independent of the ratio $R \equiv J_{z} / J_{x y}$, where $J_{z}$ and $J_{x y}$ are the interplanar and intraplanar coupling strengths respectively. This hypothesis is supported by calculations for $\gamma$ and $\nu$ for the sc and fcc Ising lattices.
\end{abstract}

The universality hypothesis [1] is designed to provide an answer to the question 'On what features of an interaction Hamiltonian do criticalpoint exponents depend ?'. The validity of the universality hypotheses has been seriously questioned by recent results [2-4] that certain exponents for a special sort of two-dimensional Ising-like model varied smoothly with the magnitude of a fourspin interaction. It is, of course, quite possible that the universality hypothesis is still valid for realistic, three-dimensional systems, and it is this problem that we address in the present work.

A large portion of the models that are of experimental interest can be described by the classical Hamiltonian

$$
\mathscr{H}=-\sum_{r, \boldsymbol{r}} \sum_{\alpha=1}^{D} J_{r-\boldsymbol{r}}^{\alpha} s_{r}^{\alpha} s_{\boldsymbol{r}^{\prime}}^{\alpha},
$$

where the vectors $r, r^{\prime}$ label sites on a $d$-dimensional lattice of $N$ sites, $S_{\boldsymbol{r}}^{\alpha}$ is the $\alpha$ th component of a spin vector in a $D$-dimensional spin space, and $J_{r-r}^{\alpha} r^{\prime}$ denotes an exchange constant. For $D=1,2,3$ and $\infty$ the Hamiltonian (1) reduces to the Ising, plane rotator, classical Heiselberg, and spherical models respectively [5].

Since current evidence favors spin independence of the critical-point exponents [6], we have considered only the classical Hamiltonian (1). Thus essentially the only parameters upon which critical-point exponents might depend are (i) the lattice dimensionality, $d$, (ii) the spin

* This work forms a portion of a Ph.D.thesis submitted to the M.I.T. Physins Denartment by Gerald Paul.

** NSF Predoctoral Fellow.

*** Supported in part by NSF Grant No.GP-15428. space dimensionality, $D$, (iii) the 'lattice anisotropy' or dependence of $J_{r-r}^{\alpha} r^{\prime}$ upon the direction of $r-r$ ', (iv) the 'spin space anisotropy' or dependence of $J_{r-r}^{\alpha}$ upon $\alpha$, and (v) the range of interaction, or dependence of $J_{\boldsymbol{r}-\boldsymbol{r}^{\prime}}^{\alpha}$ upon $\left|\boldsymbol{r}-\boldsymbol{r}^{\prime}\right|$.

The universality hypothesis would predict that critical-point exponents depend upon (ii) and (iv) only through the symmetry of the ordered phase, so that one can assign an 'effective $D$ ' to an arbitrary anisotropic interaction (as concluded [7] from high-temperature series expansions). Here we study the possible dependence of criticalpoint exponents upon (iii), lattice anisotropy; in a subsequent work we study the possible dependence upon (v), the range of interaction. Accordingly, we have used the technique of bond and vertex renormalization [8] to calculate hightemperature series expansions for the two-spin correlation function $\Gamma(\boldsymbol{r})$ for the interaction Hamiltonian

$$
\begin{aligned}
\mathscr{H}_{\text {l.anis. }} & =-\sum_{\langle i j\rangle}^{x y} J_{x y} s_{i}^{z} s_{j}^{z}-\sum_{\langle i j\rangle}^{z} J_{z} s_{i}^{z} s_{j}^{z} \\
& \equiv-J_{x y}\left[\sum_{\langle i j\rangle}^{x y} s_{i}^{z} s_{j}^{z}+R \sum_{\langle i j\rangle}^{z} s_{i}^{z} s_{j}^{z}\right]
\end{aligned}
$$

Here $R \equiv J_{z} / J_{x y}$ and the first summation is over pairs of nearest-neighbor sites whose relative displacement vcctor $\boldsymbol{r}_{i j}$ has no $z$ component, while the second summation is over all other pairs of nearest neighbor sites. We treat the simple cubic (sc) and the face centered cublc (fcc) lat tices, and we note that in the limit $R \rightarrow 0$, the sc and fcc lattices reduce to non-interacting planes $(d=2)$, while in the limit $R \rightarrow \infty$, the sc lattice reduces to an array of non-interacting chains $(d=1)$, and the fcc lattice reduces to a bcc lattice $(d=3)$. 


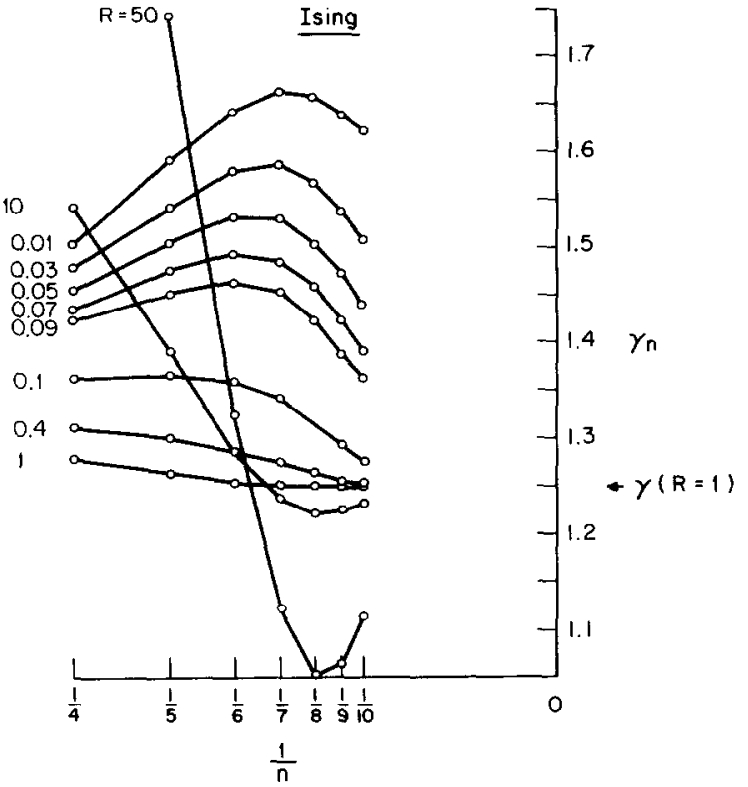

Fig.1. The dependence upon $1 / n$ of successive estimates from Park's method, for $\mathscr{H}$ lanis., sc lattice, for various values of $R \equiv J_{z} / J_{x y}$. The marked curvature displayed for $R \ll 1$ and $R \gg 1$ indicates that the series have not yet settled down to the true threedimensional behavior. The behavior shown in this figure is representative of that obtained by other extrapolation methods (see discussion in text).

To the best of our knowledge this work is the

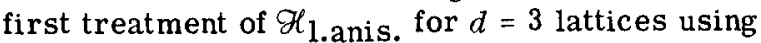
series expansions *.

We have calculated the coefficients in the high-temperature series expansion for the twospin correlation function,

$\Gamma(\boldsymbol{r})=\sum_{n=0}^{\infty} g_{n}(\boldsymbol{r}) x^{\mathrm{n}}$

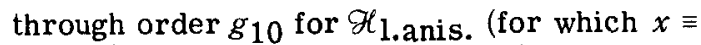
$\left.\equiv J_{x y} / k T\right)$. The coefficients $g_{n}(\boldsymbol{r})$ were then utilized to calculate series of corresponding lengths for the reduced zero-field isothermal susceptibility,

$\overline{\mathrm{X}}_{T} \equiv \sum_{\boldsymbol{r}} \Gamma(r) \equiv \sum_{n=0}^{\infty} a_{n} x^{n} \sim \epsilon^{-\gamma}$,

for the 'second moment',

* For the $d=2$ Ising model, it has been shown by various authors that $\alpha, \beta$ and $\nu$ are constant for $0<J_{x} / J_{y}<\infty$. $\mu_{2} \equiv \sum_{r} r^{2} \Gamma(r) \equiv \sum_{n=0} b_{n} x^{n} \sim \epsilon^{-(2 \nu+\gamma)}$,

and for the zero-field specific heat,

$C_{I I}=(\partial E / \partial T)_{H} \sim \epsilon^{-\alpha}$.

It is by now conventional to obtain estimates for critical-point exponents by making extrapolations based upon a finite number of exactlycalculated terms, and many methods have been proposed to render this step possible; for example

(i) the ratio mentioned, (ii) the method of Padé approximants, (iii) 'Park's method' [9], (iv) ' $T$ c renormalization' [7]. We note that methods (iii) and (iv) provide estimates of the criticalpoint exponents that do not depend in any way upon the critical temperature.

For $0.08 \leqslant R$ for the fcc lattice, and for $0.2 \leqslant R \leqslant 5.0$ for the sc lattice, both the exponents $\gamma$ and $\nu$ were found to be unchanged from their values for the isotropic case, $R=1$. For very small values of $R$ (as well as for very large values of $R$ in the case of the sc lattice), we find evidence from the series (cf., e.g., fig. 1) that ten terms are not sufficient to indicate the truc three-dimensional nature of the lattice. Since it would be difficult to imagine why the series should 'lock in' on the $R=1$ values of the exponents for all $R$ within such a wide range, and since we have clear eivdence that the series are not convergent outside the range of $R$ for which the $R=1$ values are indicated, we feel that the exponents $\gamma$ and $\nu$ probably take on their values for an isotropic lattice $(R=1)$ for all $R$ in the range $0<R<\infty$, changing discontinuously to the appropriate values for a two-dimensional lattice $(\gamma=1.75$ and $\nu=1)$ for $R=0$ (and, in the case of the sc lattice, changing discontinuously to the behavior of a one-dimensional lattice for $R=\infty) * *$.

In summary, then, we conclude that $\gamma=$ $=1.25 \pm 0.01$ and $\nu=0.635 \pm 0.010$ for $\mathscr{H}_{\text {l.anis. }}$. for the range $0.08 \leqslant R$ (fcc) and $0.2 \leqslant R \leqslant 1 / 0.2$ (sc) ***. For values of $R$ outside this range, we

** For the $C_{H}$ series none of the methods was sufficiently smoothtly convergent for us to make any firm conclusions at this time concerning the dependence of $\alpha$ upon $R$.

*** Our estimates revealed that $2 \nu$ was slightly greater than $\gamma$ for most cases, however, the confidence limits on the exponent $\eta=2-\gamma / \nu$ were sufficiently large as to preclude any statement at this time concerning whether in fact $\eta>0$. 
have evidence that our series are not sufficiently long to display their true asymptotic behavior. Therefore, our results are fully consistent with the predictions of the universality hypothesis.

We wish to thank M. Ferer, M. A. Moore and M. Wortis for providing us with the computer program they used for their nearest-neighbor isotropic calculations. We also wish to acknowledge many helpful conversations with Sava Milosević and M. Howard Lee.

\section{References}

[1] R. B. Griffiths, Phys. Rev. Letters 24 (1970) 1489; L. P. Kadanoff, in: Proc. Varenna Summer School on
Critical Phenomena, ed. M.S.Green (Academic Press, New York, 1971).

[2] R. J. Baxter, Phys. Rev. Lett. 26 (1971) 832, and to be published.

[3] F. Y. Wu, to be published.

[4] L. P. Kadanoff and F. Wegner, private communication.

[5] H. E. Stanley, Phys. Rev. Lett. 20 (1968) 589; Phys. Rev. 176 (1968) 718.

[6] For the Ising indices, see D. M. Saul, D. Jasnow and M. Wortis, to be published;

For the Heisenberg exponents, see M. H. Lee and H. E. Stanley, Phys. Rev. B4 (1971) 1613 and references contained therein.

[7] D. Jasnow and M. Wortis, Phys. Rev. 176 (1968) 739.

[8] M. A. Moore, D. Jasnow and M. Wortis, Phys. Rev. Lett. 23 (1969) 861.

[9] D. Park, Physica 22 (1956) 932 ;

A. J. Guttmann, Ph. D. Thesis, Univ, of New South Wales, 1969. 